\title{
Les soins hospitaliers aux personnes qui consomment des drogues injectables
}

\author{
Thomas D. Brothers MD, John Fraser MD, Duncan Webster MD
}

Citation : CMAJ 2021 March 22;193:E310. doi : 10.1503/cmaj.202124-f

Voir la version anglaise de l'article ici : www.cmaj.ca/lookup/doi/10.1503/cmaj.202124

1

La consommation de drogues injectables et les

hospitalisations qui en résultent sont de plus en plus fréquentes au Canada

Au Canada, le nombre de personnes qui consomment des drogues injectables a augmenté, passant de 130000 en 2011 à 171900 en $2016^{1}$. En Ontario, les hospitalisations de patients présentant une endocardite infectieuse liée aux injections ont quintuplé, passant de 34 en 1997 à 176 en $2014^{2}$.

Les personnes qui consomment des drogues injectables peuvent ne pas se sentir à l'aise de le révéler

Les professionnels de la santé devraient employer une approche sans jugement pour questionner leurs patients sur le type, la quantité et la fréquence de leur consommation de drogues. La consommation de drogues illicites est stigmatisée et criminalisée, ce qui influence les soins en milieu hospitalier ${ }^{3,4}$. Les personnes qui consomment des drogues injectables demandent des soins qui privilégient la confiance et les relations interpersonnelles, qui reconnaissent les déséquilibres de pouvoir et laissent de la place aux patients ${ }^{3}$.

\section{Débuter le traitement de la toxicomanie en centre hospitalier améliore les résultats}

Un essai clinique randomisé et contrôlé ${ }^{5}$ et plusieurs études de cohorte ${ }^{4,6}$ ont observé que l'instauration en milieu hospitalier d'un traitement par agonistes opioïdes (p. ex., la buprénorphine ou la méthadone) s'accompagnait d'une augmentation de la participation au traitement et d'une diminution des réadmissions ou des décès, comparativement aux demandes de consultation de patients externes. Tous les médecins peuvent prescrire de la buprénorphine ou de la méthadone en centre hospitalier, bien que certains organismes de réglementation provinciaux exigent une certification pour instaurer un traitement par agonistes opioïdes. Les services de consultation pour le traitement de la toxicomanie peuvent offrir de l'aide dans le cas de diagnostics complexes et de prise en charge, y compris dans le cas de troubles liés à la consommation de stimulants et à la polytoxicomanie ${ }^{4}$.
Traiter le sevrage des opioïdes de façon

efficace diminue les congés prématurés de patients contre l'avis du médecin

Les personnes qui consomment des drogues injectables mentionnent quitter les centres hospitaliers de façon prématurée en raison d'un traitement insuffisant de leur sevrage et de leur douleur ${ }^{3}$. La plupart de ces personnes consomment des opioïdes ${ }^{1}$. Les médicaments à base d'opioïdes sont beaucoup plus efficaces que ceux qui n'en contiennent pas ( $p$. ex., la clonidine) pour soulager les symptômes de sevrage des opioïdes et faciliter les soins hospitaliers ${ }^{3,4}$. Si les patients refusent le traitement par agonistes opioïdes, les médecins devraient considérer l'utilisation de doses fréquentes d'opioïdes à courte durée d'action (p. ex., de l'hydromorphone toutes les 3 heures), graduellement augmentées pour soulager les symptômes ${ }^{4}$. Traiter le sevrage ou la douleur à l'aide d'opioïdes n'aggrave pas la dépendance. Le partage du processus de décision est essentiel ${ }^{4}$.

\section{Les centres hospitaliers devraient offrir des services de réduction des préjudices} Les centres hospitaliers doivent modifier leurs politiques traditionnelles d'abstinence complète 4 . Les services de réduction des préjudices, comme le soutien des pairs, des fournitures d'injection stériles et des réceptacles pour l'élimination des déchets, des trousses de naloxone pour utilisation à domicile et des centres de consommation supervisés, devraient être offerts par les centres hospitaliers. Lorsqu'ils le sont, ces services soutenus par des données probantes contribuent à la réduction des risques d'infection et de surdose chez les patients hospitalisés4. 


\section{Références}

1. Jacka B, Larney S, Degenhardt L, et al. Prevalence of injecting drug use and coverage of interventions to prevent HIV and hepatitis $C$ virus infection among people who inject drugs in Canada. $\mathrm{Am} \mathrm{J}$ Public Health 2020;110:45-50.

2. Coverdale N, Brogly S, Payne D, et al. Rates of infective endocarditis in substance use disorder and associated costs in Ontario. Can J Addict 2019; 10:36-43

3. McCall J, Pauly B. Sowing a seed of safety: providing culturally safe care in acute care settings for people who use drugs. J Mental Health Addic Nurs 2019; 3:e1-7.

4. Allen D, Brouwer J, Dong K, et al. Management of substance use in acute care settings in Alberta: guidance document. Edmonton: Canadian Research Initiative on Substance Misuse (CRISM); 2020. Accessible ici : https://crismprairies.ca/management -of-substance-use-in-acute-care-settings-in-alberta -guidance-document/ (consulté le 4 déc. 2020).

5. Liebschutz JM, Crooks D, Herman D, et al. Buprenorphine treatment for hospitalized, opioiddependent patients: a randomized clinical trial. JAMA Intern Med 2014;174:1369-76.

6. Marks LR, Munigala S, Warren DK, et al. Addiction medicine consultations reduce readmission rates for patients with serious infections from opioid use disorder. Clin Infect Dis 2019;68: 1935-7.
Intérêts concurrents : Thomas Brothers est soutenu par une bourse de recherche de la Fondation pour la recherche en médecine interne de l'Université Dalhousie, une bourse postdoctorale Killam, une bourse commémorative Ross Stewart Smith en recherche médicale et une allocation du programme de clinicien-chercheur aux études supérieures (toutes trois de la Faculté de médecine de l'Université Dalhousie), une bourse de recherche des Instituts de recherche en santé du Canada (INRS-NRF n 171259) et une bourse de recherche du Research in Addiction Medicine Scholars (RAMS) Program (National Institute on Drug Abuse; n R25DA033211). Aucun autre intérêt concurrent n’a été déclaré.

Cet article a été révisé par des pairs.

Affiliations : Division de médecine interne générale et du programme de clinicien-chercheur (Brothers), Division des maladies infectieuses (Webster), Département de médecine, Université Dalhousie; Services de santé mobiles de rue (Fraser), Centre de santé communautaire North End, Halifax, N.-É.; Centre collaboratif pour la santé inclusive UCL (Brothers), Institut d'épidémiologie et de soins de santé, Collège universitaire de Londres, Londres, G.-B.; Division des maladies infectieuses (Webster), Hôpital régional Saint John, Saint John, N.-B.

Propriété intellectuelle du contenu : Il s'agit d'un article en libre accès distribué conformément aux modalités de la licence Creative Commons Attribution (CC BY-NC-ND 4.0), qui permet l'utilisation, la diffusion et la reproduction de tout médium à la condition que la publication originale soit adéquatement citée, que l'utilisation se fasse à des fins non commerciales (c.-à-d., recherche ou éducation) et qu'aucune modification ni adaptation n'y soit apportée. Voir : https://creativecommons.org/licenses/by-nc-nd/4.0/deed.fr.

Correspondence : Thomas Brothers, thomas.brothers@dal.ca

Le CMAJ vous invite à soumettre vos textes pour la rubrique « Cinq choses à savoir... » en ligne à http://mc.manuscriptcentral.com/cmaj. 